\title{
Soliton annihilation in the perturbed sine-Gordon system
}

\section{Pedersen, Niels Falsig; Samuelsen, Mogens Rugholm; Welner, D.}

\section{Published in:}

Physical Review B

Link to article, DOI:

10.1103/PhysRevB.30.4057

Publication date:

1984

\section{Document Version}

Publisher's PDF, also known as Version of record

Link back to DTU Orbit

Citation (APA):

Pedersen, N. F., Samuelsen, M. R., \& Welner, D. (1984). Soliton annihilation in the perturbed sine-Gordon system. Physical Review B, 30(7), 4057-4059. https://doi.org/10.1103/PhysRevB.30.4057

\section{General rights}

Copyright and moral rights for the publications made accessible in the public portal are retained by the authors and/or other copyright owners and it is a condition of accessing publications that users recognise and abide by the legal requirements associated with these rights.

- Users may download and print one copy of any publication from the public portal for the purpose of private study or research.

- You may not further distribute the material or use it for any profit-making activity or commercial gain

- You may freely distribute the URL identifying the publication in the public portal

If you believe that this document breaches copyright please contact us providing details, and we will remove access to the work immediately and investigate your claim 


\title{
Soliton annihilation in the perturbed sine-Gordon system
}

\author{
N. F. Pedersen, M. R. Samuelsen, and D. Welner \\ Physics Laboratory I, The Technical University of Denmark, \\ DK-2800 Lyngby, Denmark \\ (Received 23 April 1984)
}

\begin{abstract}
Fluxon-antifluxon annihilation in the perturbed sine-Gordon equation with loss and driving terms is investigated. For the infinite line we find a simple analytic expression for the threshold driving term corresponding to annihilation. With the application of the results to a Josephson junction of finite length an expression for the current voltage characteristic of a zero-field step is derived. The analytical results are in good agreement with numerical simulations. The method is extendable to other systems.
\end{abstract}

An important problem in soliton dynamics is the solitonantisoliton collision process. For the lossless sine-Gordon system, the problem is reduced to a calculation of the resulting phase shift, which may be done analytically. For the sine-Gordon system with loss and driving terms, the problem is more involved and requires, in addition, a calculation of the energy loss during the collision. Here, only numerical methods are available. In the present paper we have treated the latter question using perturbational methods and derived a simple analytical expression for the energy loss during the collision. This enables us to determine the threshold driving term below which a soliton and an antisoliton annihilate each other on the infinite line. The results are used to study soliton dynamics on a line of finite length. Here, the average velocity of a single fluxon is calculated. This is directly applicable to the current voltage characteristic of a zero-field step in a Josephson junction. The results presented here are general in the sense that they are applicable to other systems described by the nonintegrable sine-Gordon equation. Other perturbations than collisions may also be treated, and other systems than the sine-Gordon system may be treated as long as the solitonantisoliton solution in the lossless case is known.

The system under investigation is the perturbed sineGordon system with loss and driving (bias) terms

$$
-\phi_{x x}+\phi_{t t}+\alpha \phi_{t}+\sin \phi=\eta \text {. }
$$

This may describe, for example, a Josephson junction, a chain of coupled pendula, or the motion of a domain wall in a ferromagnetic crystal. Previous analysis ${ }^{1}$ show that the simplest nontrivial solution to Eq. (1) is a sine-Gordon soliton

$$
\phi \cong 4 \tan ^{-1} \exp (\xi)+\sin ^{-1} \eta, \quad \xi=\gamma(u)(x-u t),
$$

where $\gamma(u)=\left(1-u^{2}\right)^{-1 / 2}$ is the Lorenz factor, and $u$ is power balance velocity, $u_{\infty}$, determined by

$$
u_{\infty}=\left[1+(4 \alpha / \pi \eta)^{2}\right]^{-1 / 2} .
$$

For the unperturbed case $(\alpha=\eta=0)$ another solution is the soliton-antisoliton solution given by

$$
\phi=4 \tan ^{-1}\left(\frac{\sinh T}{u \cosh X}\right),
$$

where $T=u \gamma(u) t$ and $X=\gamma(u) x$. An investigation of Eq. (3) shows that even in the lossless case, a soliton colliding with an antisoliton will experience a phase shift $\delta$ (spatial advance) given by

$$
\delta=-2\left(1-u^{2}\right)^{1 / 2} \ln u
$$

however, no annihilation takes place.

With loss and driving terms included, we use perturbational methods ${ }^{1,2}$ to calculate the time rate of change of the energy $H$ as

$$
\frac{d H}{d t}=\int_{-\infty}^{\infty}\left(\eta \phi_{t}-\alpha \phi_{t}^{2}\right) d x .
$$

Here, the first term represents the energy delivered to the infinite line and the second term the energy dissipated. Assuming the soliton-antisoliton solution, Eq. (3), the integral may be evaluated. After some rather lengthy calculations the result is

$$
\frac{d H}{d t}=\eta 4 \pi \frac{\cosh T}{\cosh Z}-16 \alpha \gamma(u)\left(\frac{\cosh T}{\cosh Z}\right)^{2}\left(1+\frac{2 Z}{\sinh 2 Z}\right),
$$

where, for convenience, the variable $Z$, defined by $\sinh Z=(\sinh T) / u$, has been introduced. Thus, the change in energy during time $2 t$ may be written

$$
\begin{aligned}
\Delta H & =\int_{-t}^{t} \frac{d H}{d t} d t \\
& =\int_{-Z}^{Z}\left[\frac{4 \pi}{\gamma(u)} \eta-16 \alpha \gamma(u)\left(\frac{\cosh T}{\cosh Z}\right)\left(1+\frac{2 Z}{\sinh 2 Z}\right)\right] d Z .
\end{aligned}
$$

In the limit $t \rightarrow \infty$, the soliton-antisoliton solution, Eq. (5), tends to single soliton and antisoliton solutions, the velocity of which is given by the power balance velocity $u_{\infty}$ of Eq. (2). Inserting $u_{\infty}$ in Eq. (7) removes the divergence of the integral as $t$ goes to infinity. The result for the energy dissipated during the collision is then

$\Delta H=16 \alpha \int_{-\infty}^{\infty}\left(u_{\infty}-\frac{\cosh T}{\cosh Z}-\frac{(\cosh T) 2 Z}{(\cosh Z) \sinh 2 Z}\right) d Z$.

For $u=1$ one obtains

$$
\Delta H=4 \pi^{2} \alpha \text {. }
$$

For $u<1$, the integral cannot readily be evaluated by analytical methods. A numerical investigation shows that the right-hand side of Eq. (9) should be multiplied by a function $f(u)$, which decreases smoothly from 2 at $u=0$ to 
1 at $u=1$. A linear expansion of Eq. (8) around $u=1$ is possible and gives a good approximation down to $u \cong 0.5$. The result is

$$
\Delta H=4 \pi^{2} \alpha f(u) \cong 4 \pi^{2} \alpha\left(1+\frac{4}{\pi^{2}}(1-u)\right) .
$$

So far we have considered a soliton and an antisoliton on an infinite line. This problem is mathematically equivalent to having a single soliton on a semi-infinite line colliding with a boundary condition $\phi_{x}=0$ at $x=0$. In any case, the threshold driving term, $\eta_{\text {th }}$, corresponding to annihilation may be calculated by expressing that the incident soliton energy, ${ }^{1,2} E_{i}=8 \gamma\left(u_{\infty}\right)$ is equal to the energy loss [one-half of Eq. (9) for a single soliton] plus the soliton rest energy, i.e.,

$$
8 \gamma\left(u_{\infty}\right)=2 \pi^{2} \alpha+8 \gamma(0) \text {. }
$$

With the use of Eq. (2) for $u_{\infty}$, the surprisingly simple approximate result (valid for small velocities) is

$$
\eta_{\mathrm{th}} \cong(2 \alpha)^{3 / 2} \text {. }
$$

Figure 1 shows a comparison of Eq. (12) with numerical simulation (both our own and those of Ref. 1). The agreement, especially for $\alpha<0.1$, is excellent. A further question is to what extent Eq. (12) is applicable to a line of finite length. Figure 1 also shows numerical results for $l=20$ (Ref. 3) and new results for $l=10$. We observe that $\eta_{\text {th }}$ is increased typically for $\alpha l \leqslant 1$, but that Eq. (12) is applicable when $\alpha l>1$. This is reasonable, since the condition $\alpha l>1$ ensures that the soliton being slowed down from a collision has obtained the power balance velocity again before the new collision.

The dynamics of a single soliton moving on a line of finite length $l$ may be treated using the methods described in Ref. 2 by noting that the energy loss at each boundary is $2 \pi^{2} \alpha$.

The equation of motion for the soliton momentum, ${ }^{1,2}$ $P=8 u \gamma(u)$, is

$$
\frac{d P}{d t}=-\alpha P+2 \pi \eta
$$

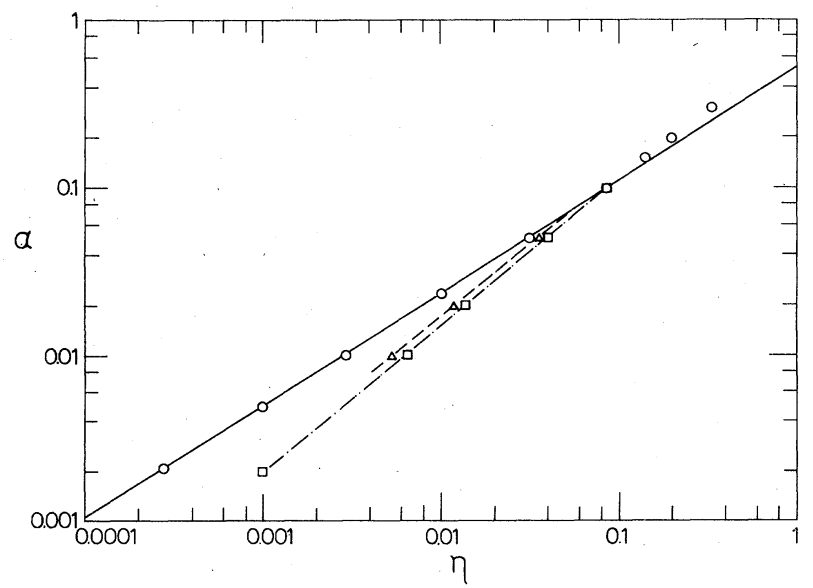

FIG. 1. Threshold annihilation curves. Circles: numerical simulation for the infinite line, full curve, theory, Eq. (12). Squares and triangles: numerical simulation for $l=10$ and $l=20$ (from Ref. 3). Dashed and dash-dotted curves are smooth curves through the data points.
The soliton trajectories are qualitatively shown in the inset of Fig. 2. Adjusting the methods of Ref. 2 to those trajectories, and using $\Delta E_{23}=\Delta E_{41}=-2 \pi^{2} \alpha$ instead of $4 \pi \kappa$, we find, after some trivial but rather lengthy calculations, that the average velocity $u_{\mathrm{av}}^{\prime}$ is given by

$$
\begin{aligned}
\frac{1}{u_{\mathrm{av}}^{\prime}}=\frac{1}{u_{\infty}}[1 & +\frac{1}{2}\left(\frac{\pi^{2}}{2 l}\right) \frac{1}{A\left(1+A^{2}\right)} \\
& \left.+\frac{1}{8}\left(\frac{\pi^{2}}{2 l}\right)^{2} \frac{\alpha l}{2 u_{\infty}} \operatorname{coth} \frac{\alpha l}{2 u_{\infty}} \frac{2+3 A^{2}}{\left[A\left(1+A^{2}\right)\right]^{2}}\right],
\end{aligned}
$$

where $A=\pi \eta / 4 \alpha$ and $\eta>(2 \alpha)^{3 / 2}$.

Note that Eq. (14) gives the average velocity calculated on the basis of the energy loss alone. The true average velocity, $u_{\mathrm{av}}$, must be corrected with the "structural" phase shift from Eq. (4). Introducing the parameter $A$ from Eq. (14) the result is

$$
u_{\mathrm{av}}=u_{\mathrm{av}}^{\prime}\left\{1-\ln \left(1+A^{-2}\right) /\left[\left(1+A^{2}\right)^{1 / 2} l\right]\right\}^{-1} .
$$

A simple improvement of Eqs. (14) and (15) may be done by retaining the velocity-dependent reflection loss $[f(u)$ from Eq. (10)]. The factor $f\left(u_{\infty}\right)$ from Eq. (10) will then appear as a factor to the expansion parameter ${ }^{4} \pi^{2} / 2 l$ in Eq. (14). Figure 2 shows a typical example $(\alpha=0.1, l=10)$ of the importance of the various contributions discussed above. It is noted that with phase-shift- and velocitydependent reflection loss included [curve (c)], the agreement with the numerical simulation (dots) is excellent. The increase in the average velocity due to the phase shift is important, and the velocity dependence of the reflection loss should be included for small values of $\eta$. A fine structure in the numerical simulation visible in Fig. 2 for low values of $\eta$ cannot be accounted for by the present theory.

There exists a somewhat qualitative and much simpler way to derive Eq. (14). According to Eq. (13), the power delivered to a line of length $l$ is $2 \pi \eta l$. For the purpose of calculating the average velocity, this power is reduced by the

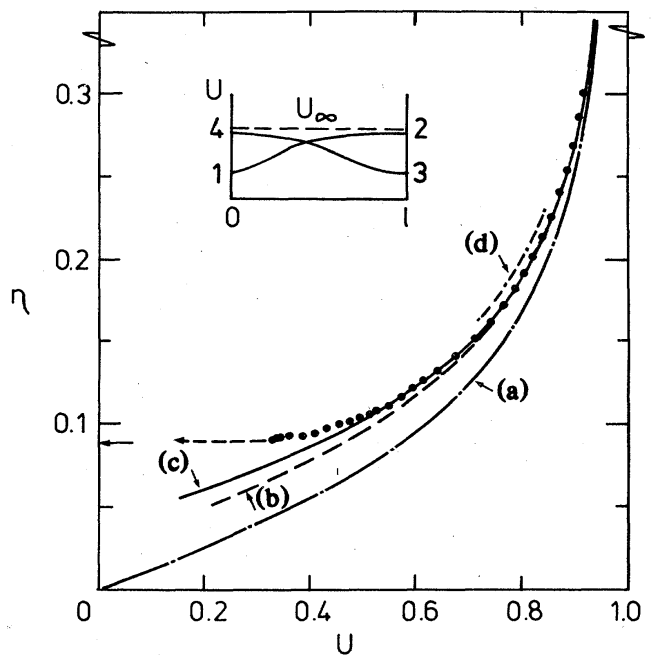

FIG. 2. Average soliton velocity, $\alpha=0.1, l=10$. Curve (a) $u_{\infty}$, curve (b) Eq. (15), curve (c) Eq. (15) with velocity-dependent damping from Eq. (10). Curve (d), curve (b) neglecting the phase shift. Dots are a full numerical simulation. The arrows correspond to $\eta_{\mathrm{th}}=(2 \alpha)^{3 / 2}$. 
loss in one end of the line which is $2 \pi^{2} \alpha$ from Eq. (9). Hence, an effective driving term, $\eta^{\prime}$, may be defined by $2 \pi \eta^{\prime} l=2 \pi \eta l-2 \pi^{2} \alpha$, i.e., $\eta^{\prime}=\eta-\pi \alpha / l$. It may be shown ${ }^{5}$ that changing the spatial distribution of the driving term gives rise to a velocity change which is only of second order in $\alpha$ as long as the total power $2 \pi \eta l$ is not changed. Hence, the average velocity may be obtained by simply substituting $\eta^{\prime}$ in Eq. (2), i.e.,

$$
u_{\mathrm{av}}^{\prime}=\left[1+\left(\frac{4 \alpha}{\pi(\eta-\pi \alpha / l)}\right)^{2}\right]^{-1 / 2}
$$

If desirable the velocity-dependent loss [Eq. (10)] may be included in $\eta^{\prime}$ and Eq. (16). It is readily verified that a second-order expansion of Eq. (16) in the quantity $\pi^{2} / 2 l$ gives a result identical to Eq. (14) except for the factor $\left(\alpha l / 2 u_{\infty}\right) \operatorname{coth}\left(\alpha l / 2 u_{\infty}\right)$ in the second-order term. (This factor is of order unity, if $\alpha l$ is small.)
Summarizing the results discussed here we may, as an example, give a simple analytic expression for the zero-field step in a Josephson junction of finite length $l$. This is directly applicable for comparison with experiments. Using Eqs. (15) and (16), the result for the (normalized) voltage is

$$
\begin{aligned}
& v=\frac{2 \pi}{l} u_{\mathrm{av}}=\frac{2 \pi}{l} \frac{\left\{1-\ln \left(1+A^{-2}\right) /\left[l\left(1+A^{2}\right)^{1 / 2}\right]\right\}^{-1}}{\left[1+\left(A-\pi^{2} / 4 l\right)^{-2}\right]^{1 / 2}}, \\
& A=\pi \eta / 4 \alpha, \quad \eta>(2 \alpha)^{3 / 2} .
\end{aligned}
$$

The results obtained here are of wider interest. First, the methods and the relatively simple result of Eq. (14) may be generalized to a nonuniform driving term, $\eta(x)$. Secondly, the method is applicable to other nonlinear systems close to a system where a soliton-antisoliton solution is known. Thirdly, in addition to the Josephson junction, the results may-possibly with small modifications-be applied to any lossy sine-Gordon system of finite size.
${ }^{1}$ D. W. McLaughlin and A. C. Scott, Phys. Rev. A 18, 1652 (1978).

${ }^{2}$ O. A. Levring, N. F. Pedersen, and M. R. Samuelsen, J. Appl. Phys. 54, 987 (1983).

${ }^{3}$ A. Davidson and N. F. Pedersen, Appl. Phys. Lett. 44, 465 (1984).

${ }^{4}$ The expansion parameter $\pi^{2} / 2 l$ is significant. For example, in a Josephson junction $l^{\prime}=\pi^{2} / 2$ is the length where the initial slope of the zero-field step is equal to the quasiparticle resistance. See, e.g., P. M. Marcus and Y. Imry, Solid State Commun. 33, 345 (1980).

${ }^{5}$ P. Bodin, N. F. Pedersen, M. R. Samuelsen, and D. Welner (unpublished). 